\title{
Dinâmica populacional de bruquíneos (Coleoptera, Chrysomelidae) em Senna multijuga (Rich.) H. S. Irwin \& Barneby (Caesalpinaceae) '
}

\author{
Lisiane Taiatella Sari ${ }^{2}$, Cibele Stramare Ribeiro-Costa ${ }^{2} \&$ James J. Roper ${ }^{3}$
}

\author{
${ }^{1}$ Contribuição número 1496 do Departamento de Zoologia, Universidade Federal do Paraná. \\ 2 Departamento de Zoologia, Universidade Federal do Paraná. Caixa Postal 19020, 81531-980 Curitiba, Paraná, Brasil. \\ Bolsista do CNPq. E-mail: lisi515@yahoo.com.br, stra@ufpr.br \\ ${ }^{3}$ Departamento de Biologia, Universidade Tuiuti do Paraná. Rua Marcelino Champagnat, 505, 80710-200, Curitiba, \\ Paraná, Brasil. E-mail: jjroper@uol.com.br
}

\begin{abstract}
Population dynamics of bruchines (Coleoptera, Chrysomelidae) in Senna multijuga (Rich.) H. S. Irwin \& Barneby (Caesalpinaceae). Senna multijuga (Rich.) H. S. Irwin \& Barneby (Caesalpinaceae) is a native ornamental tree common in southeastern Brazil and susceptible to seed damage due to bruchines. To identify seed predators and determine their population cycles, fruits from five trees were collected in 2000 and 2001 and stored in a green house until adult emerged. Seed beetles were then identified as Sennius crudelis RibeiroCosta \& Reynaud, 1998, S. puncticollis (Fåhraeus, 1839) and S. nappi Ribeiro-Costa \& Reynaud, 1998. Sennius crudelis was the most abundant species in 2000 followed by S. puncticollis and S. nappi. In 2001, Sennius crudelis was followed by S. nappi and S. puncticollis. Differences were observed among trees relating to fruiting period which reflected in the oviposition. Adult emergence begins in July in the green house and in the field. These results show that bruchine beetles have complex population dynamics, and understanding those dynamics is important to answer other ecological questions of the group.

KEY WORDS. Bruchinae, population dynamics, seed predators, Sennius.
\end{abstract}

RESUMO. Senna multijuga (Rich.) H. S. Irwin \& Barneby (Caesalpinaceae) é uma planta ornamental comum no sudeste do Brasil, suscetível ao dano por bruquíneos predadores de sementes. Com o objetivo de identificar os predadores de suas sementes e determinar seus ciclos populacionais, frutos de cinco árvores foram coletados em 2000 e 2001 e acondicionados em casa de vegetação até a emergência de adultos. Os bruquíneos foram identificados como Sennius crudelis Ribeiro-Costa \& Reynaud, 1998, S. puncticollis (Fåhraeus, 1839) e S. nappi Ribeiro-Costa \& Reynaud, 1998. Sennius crudelis foi a espécie mais abundante em 2000, seguida por S. puncticollis e S. nappi. Em 2001, Sennius crudelis foi seguida por S. nappi e S. puncticollis. Diferenças foram observadas entre as árvores com relação ao período de frutificação, refletindo na oviposição. $O$ período de emergência dos adultos se inicia em julho tanto em casa de vegetação quanto no campo. Os resultados demonstraram que as dinâmicas populacionais dos bruquíneos são complexas e a compreensão desses processos contribui para responder a muitas outras questões ecológicas do grupo.

PALAVRAS CHAVE. Bruchinae, dinâmica populacional, predadores de sementes, Sennius.

Senna multijuga (Rich.) H. S. Irwin \& Barneby (Caesalpinaceae) é uma espécie nativa utilizada na arborização de ruas, parques e jardins, principalmente na região sudeste do Brasil (LoRENZI 1992). Além disso, tem sido recomendada para utilização em projetos que visam a recuperação de áreas degradadas, pois como espécie pioneira é essencial para o sucesso de reflorestamentos, fornecendo condições necessárias ao estabelecimento de outras espécies (Bотеlнo et al. 1996). Apesar da importância potencial de $S$. multijuga, suas sementes sofrem a predação por coleópteros bruquíneos (Chrysomelidae), o que pode afetar seu poder reprodutivo. Na literatura, são citados apenas os coleópteros que ocorrem em suas sementes (JoHnson \& Siemens 1995, Casari \& Teixeira 1997, Ribeiro-Costa \& Reynaud 1998), sendo pouco o conhecimento agregado a outros parâmetros das populações ou interações com plantas hospedeiras.

Este trabalho é o primeiro de uma série resultante do estudo de diferentes aspectos da entomofauna relacionada aos frutos de Senna multijuga, no Jardim Botânico Municipal de Curitiba, Paraná. Nesta contribuição, serão reconhecidas as espécies de bruquíneos predadoras de sementes, o comportamento de oviposição de cada uma delas, bem como a dinâmica das populações. 


\section{MATERIAL E MÉTODOS}

Foram escolhidos cinco exemplares de Senna multijuga, de mesmo porte, situados no Jardim Botânico Municipal de Curitiba, Paraná, Brasil. A distância mínima entre as árvores é de 5,6 m e máxima de 10,6 m. No decorrer do texto as letras A, B, C, D e E indicam os exemplares selecionados.

As coletas foram realizadas a cada 10 dias em 2000, a partir de 31 de março, quando os frutos encontravam-se imaturos e finalizadas em 10 de outubro, quando não haviam mais frutos em duas árvores ( $D$ e E). Nas outras três ( $A, B$ e C) as coletas continuaram até 30 de novembro. Em cada coleta, foram retirados al eatoriamente 20 frutos por árvore.

As vagens das seis primeiras coletas foram mensuradas em seu maior comprimento e largura com um paquímetro e anotado o número de ovos. Em seguida, cada fruto foi preso pelo pedúnculo por barbante, individualizado em embalagem plástica inflada e mantido em casa de vegetação.

Em 2001, foram realizadas coletas de frutos com o objetivo de comparar a diversidade das espécies em dois anos consecutivos. As coletas iniciaram-se em 11 de junho, quando os frutos encontravam-se maduros, porém sem orifícios de emergência de insetos e terminaram em 8 de outubro, quando ainda haviam vagens suficientes para coleta em todos os exemplares (mais de 20 frutos). Após este período, as coletas continuaram para as árvores que ainda continham frutos ( $A$ e $E$ até o dia 28 de outubro e $B$ e $C$ até 19 de novembro).

Em interval os de 20 dias foram retirados al eatoriamente 25 frutos por planta, sendo cada lote acondicionado em frasco plástico ( $15 \mathrm{~cm} \mathrm{X} 12 \mathrm{~cm}$ ), fechado com tampa contendo abertura coberta por filó e mantido em casa de vegetação.

Frutos abertos com sementes foram col etados do chão, a partir da sétima coleta, de 8 de outubro até 9 de dezembro. As sementes com ovos de bruquíneos foram colocadas em placa de Petri, a fim de observar qual espécie de Bruchinae realiza a oviposição em sementes maduras, ainda presas às vagens.

Os frutos coletados em 2000 foram observados três vezes por semana e os colhidos em 2001 duas vezes por semana. Parte dos exemplares foram anexados à Coleção de Entomologia Pe. J.S. Moure, Departamento de Zoologia, Universidade Federal do Paraná (DZUP).

As análises estatísticas foram realizadas com base nos dados obtidos das coletas real izadas em 2000 , até 10 de outubro. $O$ programa utilizado foi o JMP, versão 3.2 (SAS INSTITUTE 1997).

Para testar se há preferência na oviposição em determinado período do desenvolvimento das vagens, procedeu-seuma análise de variância simples $(\alpha=0,05)$ e teste de Tukey, com base no número de ovos contados sobre os frutos das seis primeiras coletas de 2000. Além disso, foi realizada a Análise de Correlação de Pearson entre o comprimento das vagens e número de ovos.

Para testar a hipótese de preferência pelas fêmeas em ovipositar em determinados exemplares da planta hospedeira, re- alizou-se a análise de variância simples $(\alpha=0,05)$ e teste de Tukey, com base no número de ovos contados sobre as vagens na primeira coleta, em 31 de março de 2000.

Com o objetivo de verificar se há competição entre as espécies, foi realizada uma análise de independência usando as freqüências das espécies nas vagens. No total foram utilizados dados provenientes de 1251 vagens, ou seja, aquelas das quais emergiram bruquíneos.

Para avaliar se houve diferença entre os exemplares deS. multijuga em relação ao início do período de frutificação, foi real izada uma análise de variância simples $(\alpha=0,05)$ e teste de Tukey com os dados de comprimento e largura de 100 vagens, da primeira coleta em 2000.

\section{RESULTADOS E DISCUSSÃ O}

\section{Oviposição}

As vagens da primeira coleta, 31 de março de 2000, encontravam-se imaturas e com grande número de ovos, que foram identificados como sendo de bruquíneos, pelo aspecto morfológico externo, com filamentos nos bordos (ForISTER \& Johnson 1970, Center \& Johnson 1973, Terán \& L'ARGentier 1979, CARON et al. 2004). O comportamento de oviposição em vagens em desenvolvimento foi observado em espécies de Sennius Bridwell, 1946 (CENTER \& JOHnson 1973), e provavelmente ocorre neste período pela textura da vagem ser mais macia, o que facilita a entrada da larva de primeiro ínstar. Отт (1991) observou que as larvas de Acanthoscelides al boscutelatus (Horn, 1873) são incapazes de penetrar na parede do fruto quando estes estão parcialmente ou completamente lignificados.

Após a emergência dos adultos, constatou-se que os ovos com filamentos pertenciam a Sennius crudelis Ribeiro-Costa \& Reynaud, 1998 eS. puncticollis (Fåhraeus, 1839). Segundo FoRISTER \& Johnson (1970), os filamentos teriam a função de prevenir o desprendimento dosovos quando depositados sobre frutos imaturos, ainda em crescimento, porém RIBEIRO-CostA \& Costa (2002) não corroboraram esta idéia, pois no decorrer do tempo, verificaram que os ovos de Sennius leptophyllicola Ribeiro-Costa \& Costa, 2002 desprendiam-se da superfície da vagem.

Ovos com filamentos depositados na superfície de frutos imaturos foram observados por TERÁN \& L'ARGENTIER (1979) em Sennius laminifer (Sharp, 1885), por Center \& Johnson (1973) em Sennius morosus (Sharp, 1885), S. simulans (Schaeffer, 1907) e S. medialis (Sharp, 1885) e por RibeIRo-Costa \& Costa (2002) em Sennius leptophyllicola Ribeiro-Costa \& Costa, 2002.

A partir de maio de 2000, foram encontrados ovos sobrea superfície das vagens maduras ainda ligadas à planta-mãe, e estes ovos não apresentavam projeções filamentosas. Além disso, ovos com o mesmo aspecto foram observados sobre a superfície desementes maduras, posteriomenteà queda dos frutos no chão. Após a emergência dos adultos, foi confirmado que estes ovos pertenciam a Sennius nappi Ribeiro-Costa \& Reynaud. Da mesma forma, ovos de S. fallax (Boheman, 1839) não apresentam 
filamentos e são depositados sobre sementes maduras (CENTER \& JoHnson 1973). Com base nas guildas de oviposição de bruquíneos, recentemente revisadas por JoHnson \& Romero (2004), Sennius crudelis Ribeiro-Costa \& Reynaud, 1998, S. puncticollis (Fahråeus, 1839) eS. nappi pertencem à guilda $A$, eS. nappi à $C$, pois esta última ovipõe sobre sementes presas às vagens abertas e caídas no chão, consideradas como dispersas.

Das sementes coletadas dos frutos do chão em 2001 emergiu S. nappi. Provavelmente, esta espécie possui, no mínimo, dois períodos de oviposição, podendo ser considerada bivoltina, enquanto $\mathrm{S}$. crudelis e $\mathrm{S}$. puncticollis seriam univoltinas, pois ovipositaram apenas no início do período de frutificação. Ainda deve-se considerar a possibilidade de ter sido registrado no local outra população deS. nappi, o que dificultaria a interpretação dos resultados.

O número de ovos diminuiu progressivamente após a primeira coleta (Tab. I). Houve al ta correl ação negativa entre o comprimento das vagens e o número de ovos ( $r=-0,88)$, indicando que fêmeas de Sennius crudelis e $S$. puncticollis preferem ovipositar em vagens imaturas, em desenvolvimento, e ainda ligadas à planta-mãe.

Tabela I. Média ( \pm EP) do comprimento e largura das vagens de Senna multijuga e número médio ( $\pm E P$ ) de ovos de bruquíneos nas seis primeiras coletas de 2000 . $\mathrm{N}=100$ vagens por coleta.

\begin{tabular}{cccc}
\hline Data & Comprimento $(\mathrm{cm})$ & Largura $(\mathrm{cm})$ & Número de ovos \\
\hline $31 / 03$ & $10,27 \pm 0,22 \mathrm{a}$ & $0,85 \pm 0,04 \mathrm{a}$ & $6,53 \pm 0,84 \mathrm{a}$ \\
$10 / 04$ & $11,91 \pm 0,20 \mathrm{~b}$ & $1,27 \pm 0,02 \mathrm{~b}$ & $4,47 \pm 0,52 \mathrm{~b}$ \\
$20 / 04$ & $11,96 \pm 0,18 \mathrm{~b}$ & $1,36 \pm 0,01 \mathrm{~b}$ & $0,92 \pm 0,14 \mathrm{c}$ \\
$30 / 04$ & $12,51 \pm 0,18 \mathrm{~b}$ & $1,36 \pm 0,01 \mathrm{~b}$ & $0,83 \pm 0,18 \mathrm{c}$ \\
$10 / 05$ & $12,07 \pm 0,17 \mathrm{~b}$ & $1,37 \pm 0,01 \mathrm{~b}$ & $0,37 \pm 0,10 \mathrm{c}$ \\
$20 / 05$ & $12,33 \pm 0,20 \mathrm{~b}$ & $1,37 \pm 0,01 \mathrm{~b}$ & $0,09 \pm 0,04 \mathrm{c}$ \\
\hline
\end{tabular}

M édias seguidas de mesma letra na coluna não diferem significativamente pelo teste de Tukey $(\alpha=0,05)$. F5,594 (comprimento) $=17,26$; F5, 594 (largura) $=83,89$; F5,594 (número de ovos) $=40,51$.

No início do período de frutificação, $78 \%$ dos ovos encontravam-se aderidos às linhas de união das valvas, indicando quea postura não foi realizada al eatoriamente. TERÁN \& L'ARGENTIER (1979) também observaram que a oviposição deSennius laminifer (Sharp, 1885) é realizada nas linhas de deiscência das vagens. Este fato ocorre, provavelmente, devido à espessura mais del gada ou textura mais macia nessa região. A preferência de bruquíneos por determinados sítios de oviposição pode ser uma maneira para sobrepor as barreiras impostas pela planta hospedeira, ou mesmo uma estratégia para prevenir a mortalidade dos ovos pela ação de inimigos naturais, como parasitóides (RIBEIRO-Costa \& Costa 2002).

Em relação ao número de ovos sobre a superfície das vagens (Tab. II), os mai ores valores médios foram observados nas árvores $B$ e E, 10,40 e 12,45, respectivamente. Estas médias diferiram estatisticamente com relação às das outras árvores, mas não diferiram entre si pelo Teste de Tukey.

$O$ fato de serem encontrados mais ovos sobre as vagens das árvores $\mathrm{B}$ e E, não indica necessariamente que tenha ocorrido preferência pelas fêmeas em ovipositar nos frutos dessas árvores. Os exemplares B e E apresentaram vagens com tendência de serem, na primeira coleta, um pouco menores (Tab. II). Assim, é possível que os ovos ainda estivessem firmemente presos às mesmas na ocasião da contagem. As árvores A, C e D apresentaram frutos mais desenvolvidos emenor média de ovos. Este fato pode indicar que qualquer aumento nas dimensões das vagens, principalmente no comprimento, causaria a queda dos ovos, como citado em Ribeiro-Costa \& Costa (2002).

$A$ árvore $D$ foi a que apresentou vagens mais desenvolvidas desde a primeira coleta, e as médias de comprimento e largura das vagen s diferiram estatisticamente pelo Teste de Tukey com relação às outras árvores (Tab. II). No que se refere à largura, além da árvore $D$, a média das vagens da árvore $C$ também diferiu.

Tabela II. Média ( \pm EP) do comprimento e largura de vagens e número médio ( $\pm E P$ ) de ovos de bruquíneos da primeira coleta (31/III/2000). $\mathrm{N}=20$ vagens/árvore.

\begin{tabular}{cccc}
\hline Árvores & Comprimento $(\mathrm{cm})$ & Largura $(\mathrm{cm})$ & Número de ovos \\
\hline $\mathrm{A}$ & $10,30 \pm 0,45 \mathrm{a}$ & $0,59 \pm 0,03 \mathrm{a}$ & $3,60 \pm 1,15 \mathrm{a}$ \\
$\mathrm{B}$ & $8,85 \pm 0,36 \mathrm{a}$ & $0,62 \pm 0,06 \mathrm{a}$ & $10,40 \pm 1,76 \mathrm{~b}$ \\
$\mathrm{C}$ & $10,15 \pm 0,41 \mathrm{a}$ & $1,08 \pm 0,09 \mathrm{~b}$ & $3,40 \pm 1,17 \mathrm{a}$ \\
$\mathrm{D}$ & $12,86 \pm 0,43 \mathrm{~b}$ & $1,45 \pm 0,19 \mathrm{c}$ & $2,80 \pm 0,61 \mathrm{a}$ \\
$\mathrm{E}$ & $9,19 \pm 0,24 \mathrm{a}$ & $0,50 \pm 0,14 \mathrm{a}$ & $12,45 \pm 2,83 \mathrm{~b}$ \\
\hline Médias seguidas de mesma letra não diferem significativamente (p \\
<0.05) pelo teste de Tukey. F4, 95 (comprimento) =16,57; F4, 95 \\
(largura) = 51,75; F4, 95 (número de ovos) =7,27.
\end{tabular}

As diferenças observadas indicam que, mesmo tendo sido selecionadas árvores de mesmo porte, provavelmente com mesma idade e que receberam mesma incidência de raios luminosos, duas iniciaram o período de frutificação anteriormente às outras, ofertando prematuramente frutos para oviposi ção, podendo ocorrer inclusive maior predação de sementes nesses exemplares.

\section{Emergência de adultos}

Em 2000, os bruquíneos iniciaram a emergência a partir de julho, três meses após a primeira coleta de vagens. Nesse mesmo período, foram observados orifícios de emergência em vagens no campo, indicando que mesmo após a retirada dos frutos da planta-mãe, além de outros fatores ocasionados pelo acondicionamento dos frutos, a duração do ciclo de vida dos bruquíneos parece não ter sido afetada drasticamente em casa de vegetação.

Revista Brasileira de Zoologia 22 (1): 169-174, março 2005 
Dos frutos da primeira e segunda coletas de 2000, apesar do al to número de ovos (Tab. I), não emergiu nenhum bruquíneo, provavelmente porque os frutos encontravam-se demasiadamente imaturos e sem os recursos mínimos necessários à sobrevivência das larvas de primeiro ínstar.

Os exemplares que emergi ram das coletas seguintes pertencem à Sennius crudelis, S. puncticollis e S. nappi, que também haviam sido coletados em Senna multijuga por RibeIRo-Costa \& Reynaud (1998) em área urbana de Curitiba. Segundo Johnson (1984), Sennius Bridwell é restrito a Senna Miller, embora existam referências aos gêneros Cassia L. e Chamaecrista Moench. como plantas hospedeiras.

O número de bruquíneos registrados foi de 1535 , sendo Sennius crudelis a espécie dominante, com 1249 exemplares (81\%), seguida de S. puncticollis com 187 exemplares (12\%) e S. nappi com 99 exemplares (7\%) (Fig. 1). Em 2000 as coletas haviam cessado em outubro; entretanto, considerando os bruquíneos que emergiram dos frutos das árvores A, B e C após esta data, ou seja, até 30 de novembro, verificou-se queSennius crudel is continuou a ser a espécie dominante, com $71 \%$ do total; S. nappi passa a ser a segunda espécie dominante, 19\%, seguida de $\mathrm{S}$. puncticollis, $10 \%$.

Em 2001, o número de bruquíneos foi de 381 exemplares. As espécies foram as mesmas do ano anterior, sendo $\mathrm{S}$. crudel is, mais uma vez, a mais abundante, com 219 exemplares (58\%), seguido de S. nappi, com 134 exemplares (35\%) e S. puncticollis, com 28 exemplares (7\%) (Fig. 2). As coletas cessaram em 8 de outubro, entretanto, considerando-se os bruquíneos que emergi ram dos frutos remanescentes das outras árvores até 19 de novembro, Sennius crudelis não seria a espécie dominante, mas S. nappi, com $51 \%$ do total de bruquíneos coletados, seguida de S. crudelis, com $43 \%$ e S. puncticollis, $6 \%$. Vários fatores podem justificar a dominância de S. nappi neste segundo ano de coleta, como baixo percentual de parasitismo ou mesmo a existência de mais uma população de S. nappi .

Realizando-se a análise de independência, pode-se notar que, quando uma espécie emerge de uma vagem, outra tem menor probabilidade de emergir da mesma vagem (S. crudelis $X$ S. puncticollis, $G=31,65 ; G L=1 ; p<0,05 ; S$. crudelis $X S$. nappi, $G=145,42 ; G L=1 ; p<0,05 ;$ S. puncticollis $X$ S. nappi, $G$ $=7,06 ; G L=1 ; p<0,05)$. Isso pode ser um indicativo de que haja competição entre as espécies, tanto entre fêmeas disputando sítios de oviposição, como larvas competindo pelo recurso alimentar dentro de uma mesma semente. A existência de competição intraespecífica em bruquíneos já havia sido comentada por Janzen (1971) e PARnell (1966). Este último autor constatou ainda a competição interespecífica entre Bruchidius ater (Marsham, 1802) e um curculionídeo.

Observando-se a emergência dos bruquíneos nas diferentes árvores em 2000 (Fig. 3), pode-se notar que todas as espécies emergiram das vagens de todas as árvores. As árvores $\mathrm{B}$ eE, que apresentaram maior número de ovos com filamentos nas seis primeiras coletas, representando ovos deS. crudelis eS. puncticollis, foram as árvores das quais emergiu maior número destas duas espécies. A árvore D foi a exceção, com 501 exemplares de S. crudelis. Nesta, obteve-se a maior média de comprimento defrutos na primeira coleta, diferindo significativamente das outras, levando a crer que muitos ovos já teriam se desprendido anteriormente à contagem e que esta foi a primeira árvore a ofertar frutos. Neste caso, pode-se considerar ainda que S. crudel is mostra acentuada preferência por vagens imaturas.

No ano de 2000 , S. crudelis foi dominante em todas as árvores; em 2001 (Fig. 4) esta espécie foi mais abundante apenas em A e B. Observando-se a emergência em 2001, nota-se que $S$. puncticollis não foi coletada na árvore $E$.

Com relação aos períodos de emergência, estes são relativos aos frutos coletados até 10 de outubro de 2000 e 8 de outubro de 2001 (Figs 5 e 6).

No ano de 2000, Sennius crudelis iniciou a emergência em julho, tendo atingido o pico em setembro, com 661 exemplares. Sennius puncticollis emergiu na mesma época e atingiu o pico de emergência um mês antes, com 67 exemplares. Sennius nappi começou a emergir a partir de agosto e o pico foi no mês de outubro, com baixo número de exemplares, 43 (Fig. 5).

Em 2001, S. crudelis e S. puncticollis emergiram em julho e al cançaram o pico de emergência já no final do mesmo mês, com 139 e 19 exemplares, respectivamente. Sennius nappi começou a emergir em julho e o pico de emergência ocorreu em novembro, com 49 exemplares (Fig. 6).

Mesmo considerando-se a metodologia de coleta diferente entre os anos, observa-se que $\mathrm{S}$. crudelis e $\mathrm{S}$. puncticollis foram as mais abundantes entre jul ho e setembro. Sennius nappi com picos subseqüentes em outubro (2000) enovembro (2001).

Observando-se os períodos de emergência, cogita-se que as espécies devem ter desenvolvido estratégias de sobrevivência para aguardarem a oferta de frutos do próximo período reprodutivo de S. multijuga. TERÁN \& L'ARGENTIER (1979) observaram que os adultos de Sennius laminifer começaram a emergir de Cassia carnaval Speg. (Caesal pinaceae) no inverno e refugiaram-se nos frutos abertos de Bixa orellana L. (Bixaceae) ou no interior de folhas de Abutilon Mill. (Malvaceae), en roladas por larvas minadoras.

Dos frutos coletados após o dia 10 de outubro em 2000 das árvores A, B eC, emergiram 232 exemplares de Sennius nappi durante os meses de novembro e dezembro de 2000 e janeiro de 2001. Pelo fato de S. nappi continuar a ocorrer nessas árvores, o período de maior emergência dessa espécie é deslocado para os meses posteriores a outubro no ano de 2000 , de forma semel hante à curva obtida em 2001.

No ano de2001, dos frutos remanescentes coletados após o dia 8 de outubro, surgiu S. nappi, com 126 exemplares, durante os meses de novembro, dezembro e janeiro.

Como os bruquíneos predadores de $\mathrm{S}$. multijuga preferem realizar a oviposição no início do período de frutificação, e, ainda uma das espécies, S. nappi, ovipõe em vagens maduras 

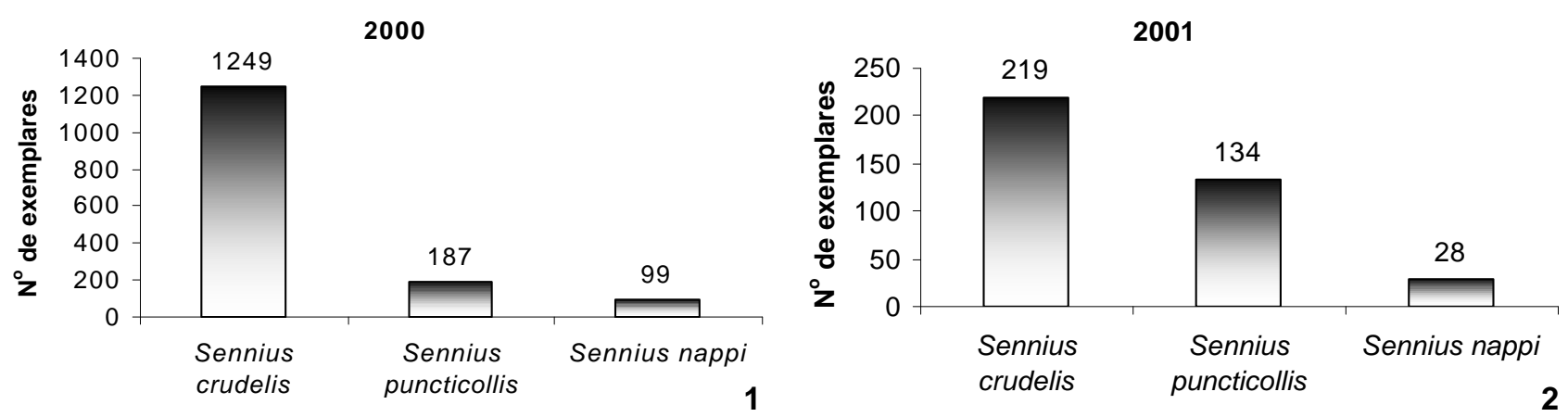

Figuras 1-2. Número de bruquíneos que emergiram de frutos de Senna multijuga em casa de vegetação: (1) ano de 2000; (2) ano de 2001.
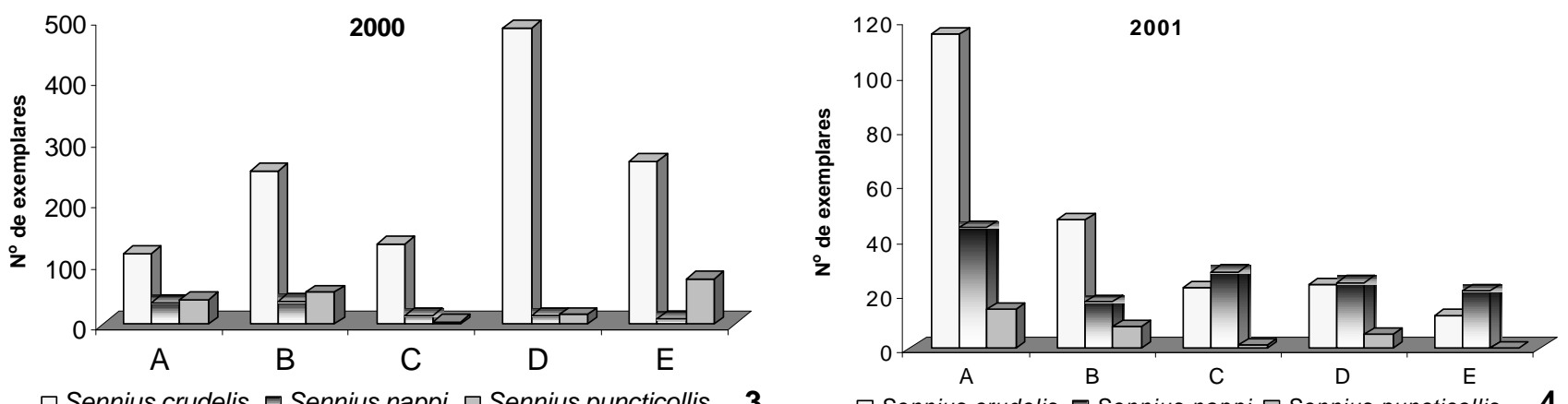

Figuras 3-4. Número de bruquíneos que emergiram em casa de vegetação, dos frutos de cinco exemplares de Senna multijuga: (3) ano de 2000; (4) ano de 2001.
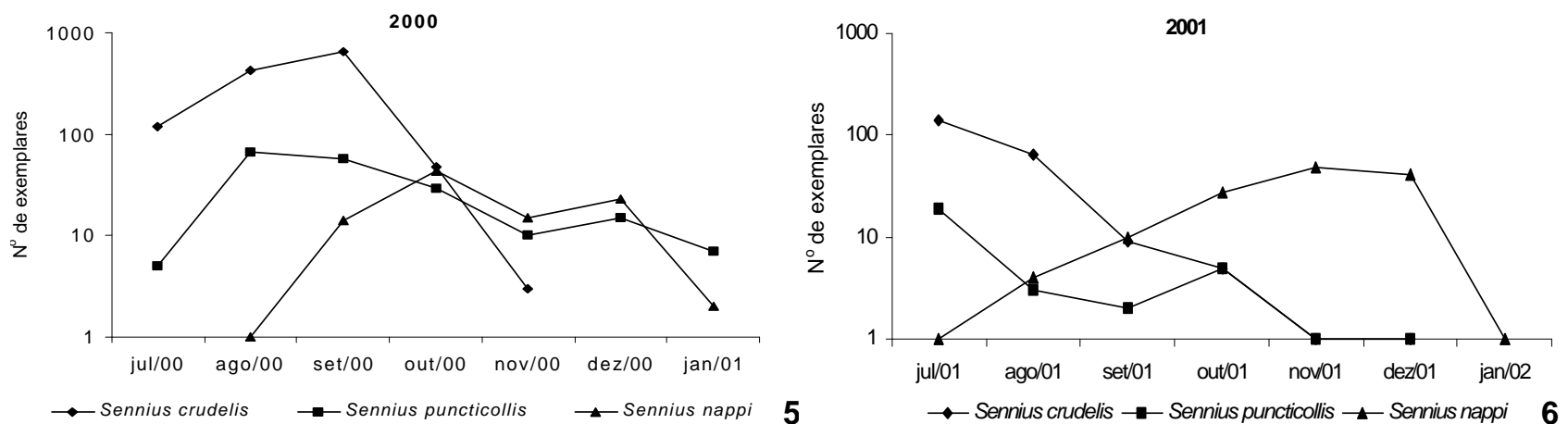

Figuras 5-6. Emergência de bruquíneos a partir de frutos de Senna multijuga em casa de vegetação (valores em escala logarítmica, $n+1$ ): (5) ano de 2000; (6) ano de 2001.

e sementes já dispersas, várias barreiras deS. multijuga contra o ataque de predadores já foram sobrepostas. Sennius crudelis eS. puncticollis ovipositam nas vagens imaturas e desenvolvem-se juntamente com as sementes. Desta forma, estas espécies devem apresentar vantagens, pois as larvas e pupas estão protegidas dentro dos frutos, completando seu desenvolvimento e garantindo o sucesso reprodutivo. Sennius nappi, ao infestar os frutos tardiamente, apresenta potencial para sobrepor as barreiras físicas impostas pela dureza das vagens maduras e do tegumento das sementes.

Os resultados deste estudo sugerem que as várias espécies de bruquíneos podem interagir e influenciar no sucesso reprodutivo deS. multijuga, e sua utilização deve levar em conta o impacto da predação de suas sementes. 


\section{AGRA DECIMENTOS}

A Gert Hatschbach, Prefeitura Municipal de Curitiba e Sandro Menezes, Fundação O Boticário, pela identificação da planta hospedeira.

\section{REFERÊNCIAS BIBLIOGRÁ FICAS}

Botelho, S.A.; A.C. Davide \& J.M.R. FariA. 1996. Desenvolvimento inicial de seis espécies florestais nativas em dois sítios, na região Sul de Minas Gerais. Revista Cerne, Lavras, 2 (1): 413.

Caron, E.; C.S. Ribeiro-Costa \& A. Linzmeier. 2004. The egg morphology of some species of Sennius Bridwell (Coleoptera: Chrysomelidae: Bruchinae) based on scanning electron micrographs. Zootaxa, Auckland, 556: 1-10.

CASARI, A.S. \& E.P. TEIXEIRA. 1997. Descriptions and biological notes of final larval instar and pupa of some seed beetles (Coleoptera: Bruchidae). Annales de la Société Entomologique de France, Paris, 33 (3): 295-321.

Center, T. \& C.D. Johnson. 1973. Comparative life histories of Sennius (Coleoptera: Bruchidae). Environmental Entomology, Lanham, 2 (4):669-672.

ForIsteR, G.W. \& C.D. JoHnson. 1970. Bionomics of M erobruchus julianus (Coleoptera: Bruchidae). Coleopterists Bulletin, Sacramento, 24 (3): 84-87.

JANZEN, D.H. 1971. Escape of Cassia grandis L. beans from predators in time and space. Ecology, Washington, 52: 964-979.

Johnson, C.D. 1984. Sennius yucatan, sp. nov., a redescription of S. infractus, and new host records for other Sennius (Coleoptera: Bruchidae). Annals of the Enthomological Society of America, Lanham, 77 (1): 56-64.

Johnson, C.D. \& J. Romero. 2004. A review of evolution of ovi- position guilds in the Bruchidae (Coleoptera). Revista Brasileira de Entomologia, Curitiba, 48 (3): 404-408.

Johnson, C.D. \& D.H. Siemens. 1995. New host records from ECuador and Venezuela for the genus Acanthoscelides (Coleoptera: Bruchidae). J ournal of Stored Products Research, Kidlington, 31 (3): 267-269.

LoRENZI, H. 1992. Árvores Brasil eiras: manual de identificação e cultivo de plantas arbóreas nativas do Brasil. Nova Odessa, Plantarum, 368p.

OTT, J.R. 1991. The biology of Acanthoscelides alboscutelatus (Coleoptera: Bruchidae) on its host plant, Ludwigia alternifolia (L.) (Onagraceae). Proceedings of the Entomological Society of Washington 93 (43): 641-651.

PARNeLL, J.R. 1966. Observations on the population fluctuations and life histories of the beetles Bruchidius ater and Apion fuscirostre on broom (Sarothamnus scoparius). Journal of Animal Ecology, Oxford, 35: 157-188.

Ribeiro-Costa, C.S. \& A.S. Costa. 2002. Comportamento de oviposição de Bruchidae (Coleoptera) predadores de sementes de Cassia leptophylla Vogel (Caesalpinaceae), morfologia dos ovos e descrição de uma nova espécie. Revista Brasileira de Zoologia, Curitiba, 19 (1): 305-316.

Ribeiro-Costa, C.S. \& D.T. Reynaud. 1998. Bruchids from Senna multijuga (Rich) I. \& B. (Caesal pinaceae) in Brazil with descriptions of two new species. Coleopterists Bulletin, Sacramento, 52 (3): 245-252

SAS INSTITUTE. 1997. JMP, version 3.2. SASInstitute Inc., Durham.

TerÁn, A.L. \& S.M. L'ARgentier. 1979. Observaciones sobre Bruchidae (Coleoptera) del noroeste argentino. II. Estudios morfológicos y biológicos de algunas espécies de Amblycerinae y Bruchinae. Acta Zoologica Lilloana, San Miguel deTucuman, 35 (1): 435-474.

Recebido em 19.X.2004; aceito em 02.II.2005. 